\title{
ORTHOGONAL POLYNOMIALS FOR THE OSCILLATORY-GEGENBAUER WEIGHT
}

\section{Gradimir V. Milovanović, Aleksandar S. Cvetković, and Zvezdan M. Marjanović}

\begin{abstract}
This is a continuation of our previous investigations on polynomials orthogonal with respect to the linear functional $\mathcal{L}: \mathcal{P} \rightarrow \mathbb{C}$, where $\mathcal{L}=\int_{-1}^{1} p(x) d \mu(x), d \mu(x)=\left(1-x^{2}\right)^{\lambda-1 / 2} \exp (i \zeta x) d x$, and $\mathcal{P}$ is a linear space of all algebraic polynomials. Here, we prove an extension of our previous existence theorem for rational $\lambda \in(-1 / 2,0]$, give some hypothesis on three-term recurrence coefficients, and derive some differential relations for our orthogonal polynomials, including the second order differential equation.
\end{abstract}

\section{Introduction}

In this paper we continue our investigation on orthogonality with respect to the oscillatory weight functions studied in $[\mathbf{7}],[\mathbf{8}]$, and $[\mathbf{9}]$. We are concerned with the following measure $d \mu(x)=\left(1-x^{2}\right)^{\lambda-1 / 2} \exp (i \zeta x) d x$, supported on the interval $[-1,1]$, where $\lambda>-1 / 2$ and where $\zeta \in \mathbb{R} \backslash\{0\}$. Evidently, the corresponding weight function

$$
w(x)=w^{\zeta, \lambda}(x)=\left(1-x^{2}\right)^{\lambda-1 / 2} \exp (i \zeta x)
$$

depends on two real parameters $\lambda$ and $\zeta$. We investigate the questions connected with the existence of polynomials $\pi_{n}$ orthogonal with respect to the linear functional

$$
\mathcal{L}[p]=\int_{-1}^{1} p(x) d \mu(x), \quad p \in \mathcal{P},
$$

where $\mathcal{P}$ is the space of all algebraic polynomials. As it has been proved in $[\mathbf{9}]$ it is enough to consider only the case $\zeta>0$, and therefore we continue our discussion only for such values of $\zeta$. As we proved in $[\mathbf{9}]$ it can be easily inferred that the orthogonal polynomials $\pi_{n}$ exist in the case $\lambda \in \mathbb{Q}$ with $\lambda>0$ and $\zeta$ is positive zero of the Bessel function of the first kind $J_{\lambda-1}$. The crucial ingredient in the proof is the result which states that non-trivial zeros of Bessel functions of the first kind are

2000 Mathematics Subject Classification: Primary 30C10; Secondary 33C47.

The authors were supported in part by the Serbian Ministry of Science (Project: Orthogonal Systems and Applications, grant number \#144004) and the Swiss National Science Foundation (SCOPES Joint Research Project No. IB7320-111079 "New Methods for Quadrature"). 
transcendental provided their index is rational. In this paper we extend the results from [9] for $-1 / 2<\lambda \leqslant 0, \lambda \in \mathbb{Q}$, and $\zeta$ being the zero of the Bessel function $J_{\lambda-1}$.

Also, in this paper, we investigate the possibility of computation of three-term recurrence coefficients for the given sequence of orthogonal polynomials $\left\{\pi_{n}\right\}_{n \in \mathbb{N}_{0}}$, as well as some consequences of the weight function $w$ being semiclassical. We also find the second order differential equation for the corresponding orthogonal polynomials.

\section{Existence of orthogonal polynomials}

According to (1.1), the moments

$$
\mu_{k}=\mathcal{L}\left[x^{k}\right]=\int_{-1}^{1} x^{k}\left(1-x^{2}\right)^{\lambda-1 / 2} \exp (i \zeta x) d x, \quad k \in \mathbb{N}_{0},
$$

can be expressed in terms of Bessel functions $J_{\nu}$ of the order $\nu$ defined by

$$
J_{\nu}(z)=\sum_{m=0}^{+\infty} \frac{(-1)^{m}(z / 2)^{\nu+2 m}}{m ! \Gamma(\nu+m+1)} .
$$

Using the initial conditions

$$
\begin{aligned}
& \mu_{0}=A J_{\lambda}(\zeta), \quad \mu_{1}=i A\left(\frac{2 \lambda}{\zeta} J_{\lambda}(\zeta)-J_{\lambda-1}(\zeta)\right) \\
& \mu_{2}=A\left[\left(1-\frac{2 \lambda(2 \lambda+1)}{\zeta^{2}}\right) J_{\lambda}(\zeta)+\frac{2 \lambda+1}{\zeta} J_{\lambda-1}(\zeta)\right]
\end{aligned}
$$

where $A=(2 / \zeta)^{\lambda} \sqrt{\pi} \Gamma(\lambda+1 / 2)$, we can see that the moments (2.1) satisfy the following recurrence relation

$$
\mu_{k+2}=-\frac{k+2 \lambda+1}{i \zeta} \mu_{k+1}+\mu_{k}+\frac{k}{i \zeta} \mu_{k-1} .
$$

The following result is proved in $[\mathbf{9}]$.

THEOREM 2.1. The moments $\mu_{k}$ can be expressed in the form

$$
\mu_{k}=\frac{A}{(i \zeta)^{k}}\left(P_{k}^{\lambda}(\zeta) J_{\lambda}(\zeta)+Q_{k}^{\lambda}(\zeta) J_{\lambda-1}(\zeta)\right), \quad k \in \mathbb{N}_{0}
$$

where $A=(2 / \zeta)^{\lambda} \sqrt{\pi} \Gamma(\lambda+1 / 2)$, and $P_{k}^{\lambda}$ and $Q_{k}^{\lambda}$ are polynomials in $\zeta$, which satisfy the following four-term recurrence relation

$$
y_{k+2}=-(k+2 \lambda+1) y_{k+1}-\zeta^{2} y_{k}-k \zeta^{2} y_{k-1},
$$

with the initial conditions

$$
P_{0}^{\lambda}(\zeta)=1, P_{1}^{\lambda}(\zeta)=-2 \lambda, P_{2}^{\lambda}(\zeta)=2 \lambda(2 \lambda+1)-\zeta^{2}
$$

and

respectively.

$$
Q_{0}^{\lambda}(\zeta)=0, Q_{1}^{\lambda}(\zeta)=\zeta, Q_{2}^{\lambda}(\zeta)=-(2 \lambda+1) \zeta
$$

Some obvious properties of the polynomials $P_{n}^{\lambda}$ and $Q_{n}^{\lambda}, n \in \mathbb{N}_{0}$, are stated in the following lemma. 
Lemma 2.1. For the degree of the polynomials $P_{n}^{\lambda}$ and $Q_{n}^{\lambda}$ we have

$$
\operatorname{deg}\left(P_{2 n}^{\lambda}, P_{2 n+1}^{\lambda}\right)=2 n \quad\left(n \in \mathbb{N}_{0}\right) \quad \text { and } \quad \operatorname{deg}\left(Q_{2 n}^{\lambda}, Q_{2 n-1}^{\lambda}\right)=2 n \quad(n \in \mathbb{N}) .
$$

The free terms in the polynomials $P_{n}^{\lambda}$ and $Q_{n}^{\lambda}$ are $(-1)^{n}(2 \lambda)_{n}$ and zero, respectively. The free term in the polynomial $Q_{n}(\zeta) / \zeta, n \in \mathbb{N}$, equals $(-1)^{n-1}(2 \lambda+1)_{n-1}$.

The leading coefficients in the polynomials $P_{2 n}^{\lambda}, P_{2 n-1}^{\lambda}, Q_{2 n+1}^{\lambda}$ and $Q_{2 n}^{\lambda}$ are $(-1)^{n},(-1)^{n}(2 n \lambda+n-1),(-1)^{n}$ and $(-1)^{n} n(2 \lambda+1)$, respectively.

Now we are ready to state and prove a stronger version of the existence theorem than the one given in $[\mathbf{9}]$.

TheOREM 2.2. Let $\lambda \in \mathbb{Q}, \lambda>-1 / 2$ and let $\zeta \neq 0$ be a zero of the Bessel function $J_{\lambda-1}$. Then the sequence of monic polynomials $\left\{\pi_{n}\right\}_{n \in \mathbb{N}_{0}}$ orthogonal with respect to the linear functional (1.1) exists.

Proof. As in $[\mathbf{9}]$ our result is based upon completely algebraic facts. Consider the sequence of Hankel determinants defined by

$$
\Delta_{n}=\left|\begin{array}{cccc}
\mu_{0} & \mu_{1} & \cdots & \mu_{n-1} \\
\mu_{1} & \mu_{2} & \cdots & \mu_{n} \\
\vdots & \vdots & \ddots & \vdots \\
\mu_{n-1} & \mu_{n} & \cdots & \mu_{2 n-2}
\end{array}\right|, \quad n \in \mathbb{N} .
$$

The sequence of orthogonal polynomials $\left\{\pi_{n}\right\}_{n \in \mathbb{N}_{0}}$ with respect to $\mathcal{L}$ exists provided $\Delta_{n} \neq 0, n \in \mathbb{N}$ (see [4]). Thus, let $\zeta$ be a nontrivial zero of the Bessel function $J_{\lambda-1}$. Then we can express the moments of the linear functional $\mathcal{L}$ in the following form

$$
\mu_{k}=\frac{A}{(i \zeta)^{k}} P_{k}^{\lambda}(\zeta) J_{\lambda}(\zeta), \quad k \in \mathbb{N}_{0}
$$

Obviously, from the Hankel determinant we can factor $\left(A J_{\lambda}(\zeta)\right)^{n+1} /(i \zeta)^{n(n+1)}$ and we are left with the sequence of Hankel determinants $H_{n}, n \in \mathbb{N}$, made from the new moment sequence $\mu_{k}^{\prime}=P_{k}^{\lambda}(\zeta), k \in \mathbb{N}_{0}$,

$$
H_{n}=\left|\begin{array}{cccc}
P_{0}^{\lambda}(\zeta) & P_{1}^{\lambda}(\zeta) & \ldots & P_{n-1}^{\lambda}(\zeta) \\
P_{1}^{\lambda}(\zeta) & P_{2}^{\lambda}(\zeta) & \ldots & P_{n}^{\lambda}(\zeta) \\
\vdots & \vdots & & \vdots \\
P_{n-1}^{\lambda}(\zeta) & P_{n}^{\lambda}(\zeta) & \ldots & P_{2 n-2}^{\lambda}(\zeta)
\end{array}\right|
$$

Accordingly, all $H_{n}, n \in \mathbb{N}$, are polynomials in $\zeta$ which have rational coefficients since $\lambda \in \mathbb{Q}$. If some $H_{n}=0$, then $\zeta$ is the zero of the polynomial $H_{n}$ with rational coefficients and $\zeta$ is zero of the Bessel function $J_{\lambda-1}$. According to [13, p. 220], based on the results by $[\mathbf{1 1}],[\mathbf{1 2}]$ (see also [6]), non-trivial zeros of the Bessel functions $J_{\lambda}, \lambda \in \mathbb{Q},-\lambda \notin \mathbb{N}$, are transcendental and cannot be the zeros of the polynomials with rational coefficients unless polynomials are identically equal to zero.

We have to prove that determinants $H_{n}$ are not identically equal to zero. To prove this fact we emphasize that, according to Lemma 2.1, the free coefficient in the polynomial $P_{n}^{\lambda}$ equals $(-1)^{n}(2 \lambda)_{n}, n \in \mathbb{N}_{0}$, so that the free coefficient in the 
polynomial $H_{n}$ equals to the value of the Hankel determinant $\widetilde{H}_{n}$ made for the sequence of moments $\mu_{k}^{\prime \prime}=(2 \lambda)_{k}, k \in \mathbb{N}_{0}$. For $\lambda>0$, we can easily recognize the Hankel determinants $\widetilde{H}_{n}$ as being the same, up to multiplicative factor $(\Gamma(2 \lambda))^{n}$, as the Hankel determinants for the generalized Laguerre weight $x^{2 \lambda-1} e^{-x}$ on $[0,+\infty)$. Hence, none of the determinants $\widetilde{H}_{n}$ cannot be equal according to the existence of the sequence of orthogonal polynomials with respect to the Laguerre weight function. This establishes the proof for $\lambda>0$ (cf. [9]).

Actually, we can calculate the determinant $\widetilde{H}_{n}$ for $\lambda>0$ in the form

$$
\widetilde{H}_{n}=\prod_{l=0}^{n-1}(n-l) ! \prod_{i=0}^{l}(2 \lambda+l-i), \quad n \in \mathbb{N} .
$$

We easily inspect that the same result about the values of the Hankel determinants $\widetilde{H}_{n}$ holds even in the case of $-1 / 2<\lambda \leqslant 0$. This due to the fact that Hankel determinants $\widetilde{H}_{n}$ as being polynomials are analytic functions of $\lambda$ as well as expressions representing their values. Inspecting values of the determinants $\widetilde{H}_{n}, n \in \mathbb{N}$, we easily conclude that $\widetilde{H}_{n} \neq 0, n \in \mathbb{N}$, for $\lambda \neq 0$. This in turn implies that $\Delta_{n} \neq 0$, $n \in \mathbb{N}$. Case $\lambda=0$ requires a special attention.

Consider now the case $\lambda=0$. As we easily inspect we have that $\widetilde{H}_{n}=0, n \in \mathbb{N}$, for $\lambda=0$. Consider determinants $H_{n}$, we have $H_{n}=(\Gamma(2 \lambda))^{n} \widetilde{H}_{n}, n \in \mathbb{N}$. Since $H_{n}, n \in \mathbb{N}$, are analytic functions of $\lambda$ we can compute values of $H_{n}, n \in \mathbb{N}$, for $\lambda=0$, as the limiting values of the expressions on the right hand side. We have

$$
\lim _{\lambda \rightarrow 0}(\Gamma(2 \lambda))^{n} \prod_{l=0}^{n-1}(n-l) ! \prod_{i=0}^{l}(2 \lambda+l-i)=n ! \prod_{l=1}^{n-1}(n-l) ! \prod_{i=0}^{l-1}(l-i) \neq 0 .
$$

Thus, we have proved that if $\lambda \in \mathbb{Q}, \lambda>-1 / 2$, and $\zeta$ is the zero of the Bessel function $J_{\lambda-1}$, then the sequence of Hankel determinants

$$
\Delta_{n}=\left(A J_{\lambda}(\zeta)\right)^{n} /(i \zeta)^{n(n-1)} H_{n}, \quad n \in \mathbb{N},
$$

contains no zero entry. Accordingly, the sequence of orthogonal polynomials with respect to the linear functional $\mathcal{L}$ exists.

Regarding Theorem 2.2, we suppose such parameters $\lambda$ and $\zeta$ which provide the existence of orthogonal polynomials $\pi_{n}$ with respect to the linear functional (1.1). The quasi inner-product $(p, q):=\mathcal{L}[p q]$, in our case has the property $(z p, q)=(p, z q)$, and because of that, the corresponding (monic) polynomials $\left\{\pi_{n}\right\}_{n \in \mathbb{N}_{0}}$ satisfy the fundamental three-term recurrence relation

$$
\pi_{n+1}(x)=\left(x-i \alpha_{n}\right) \pi_{n}(x)-\beta_{n} \pi_{n-1}(x), \quad n \in \mathbb{N},
$$

with $\pi_{0}(x)=1, \pi_{-1}(x)=0$. The recursion coefficients $\alpha_{n}$ and $\beta_{n}$ can be expressed in terms of Hankel determinants as (cf. [9]).

$$
\begin{aligned}
i \alpha_{n} & =\frac{\Delta_{n+1}^{\prime}}{\Delta_{n+1}}-\frac{\Delta_{n}^{\prime}}{\Delta_{n}}=\frac{1}{i \zeta}\left(\frac{H_{n+1}^{\prime}}{H_{n+1}}-\frac{H_{n}^{\prime}}{H_{n}}\right) \\
\beta_{n} & =\frac{\Delta_{n+1} \Delta_{n-1}}{\Delta_{n}^{2}}=\frac{1}{(i \zeta)^{2}} \frac{H_{n+1} H_{n-1}}{H_{n}^{2}}
\end{aligned}
$$


where $H_{n}$ is defined before and $\Delta_{n}^{\prime}$ is the Hankel determinant $\Delta_{n+1}$ with the penultimate column and the last row removed. The corresponding determinant $H_{n}^{\prime}$ is given by

$$
H_{n}^{\prime}=\left|\begin{array}{ccccc}
P_{0}^{\lambda}(\zeta) & P_{1}^{\lambda}(\zeta) & \ldots & P_{n-2}^{\lambda}(\zeta) & P_{n}^{\lambda}(\zeta) \\
P_{1}^{\lambda}(\zeta) & P_{2}^{\lambda}(\zeta) & \ldots & P_{n-1}^{\lambda}(\zeta) & P_{n+1}^{\lambda}(\zeta) \\
\vdots & \vdots & & \vdots & \vdots \\
P_{n-1}^{\lambda}(\zeta) & P_{n}^{\lambda}(\zeta) & \ldots & P_{2 n-3}^{\lambda}(\zeta) & P_{2 n-1}^{\lambda}(\zeta)
\end{array}\right|
$$

Although $\beta_{0}$ can be arbitrary, as usual it is convenient to take $\beta_{0}=\mu_{0}=A J_{\lambda}(\zeta)$.

In this case, however, the values of Hankel determinants cannot be found easily, but, it is clear that the recursion coefficients are rational functions in $\zeta$. Using our software package [5] we can generate coefficients even in symbolic form for some reasonable small values of $n$ (for $n \leqslant 2$ see Table 1 ).

TABLE 1. Recursion coefficients $\alpha_{n}$ and $\beta_{n}$ for $n \leqslant 2$

\begin{tabular}{|c|c|c|}
\hline$n$ & $\alpha_{n}$ & $\beta_{n}$ \\
\hline 0 & $\frac{2 \lambda}{\zeta}$ & $\left(\frac{2}{\zeta}\right)^{\lambda} \sqrt{\pi} \Gamma\left(\lambda+\frac{1}{2}\right) J_{\lambda}(\zeta)$ \\
\hline 1 & $\frac{4 \lambda(1+\lambda)-\zeta^{2}}{\zeta\left(2 \lambda-\zeta^{2}\right)}$ & $\frac{\zeta^{2}-2 \lambda}{\zeta^{2}}$ \\
\hline 2 & $\frac{(7+6 \lambda) \zeta^{6}-4 \lambda(4+\lambda)(3+2 \lambda) \zeta^{4}+32 \lambda^{2}(3+2 \lambda) \zeta^{2}-32 \lambda^{3}(2+\lambda)}{2 \zeta\left(\zeta^{2}-2 \lambda\right)\left[\zeta^{4}-\lambda(5+2 \lambda) \zeta^{2}+4 \lambda^{2}\right]}$ & $-\frac{2(1+2 \lambda)\left[\zeta^{4}-\lambda(5+2 \lambda) \zeta^{2}+4 \lambda^{2}\right]}{\zeta^{2}\left(-2 \lambda+\zeta^{2}\right)^{2}}$ \\
\hline
\end{tabular}

According to symbolic calculations we can conjecture that $H_{n}$ is a polynomial in $\zeta^{2}$ of degree $m$,

$$
H_{n}=S_{m}\left(\zeta^{2}\right)=\sum_{\nu=0}^{m} \gamma_{\nu}(\lambda) \zeta^{2 \nu}
$$

where $m=n^{2} / 4$ for even $n$ and $m=\left(n^{2}-1\right) / 4$ for odd $n$, so that, because of $(2.5)$,

$$
\beta_{2 k}=-\frac{S_{k(k+1)}\left(\zeta^{2}\right) S_{k(k-1)}\left(\zeta^{2}\right)}{\zeta^{2} S_{k^{2}}\left(\zeta^{2}\right)^{2}}, \quad \beta_{2 k+1}=-\frac{S_{(k+1)^{2}}\left(\zeta^{2}\right) S_{k^{2}}\left(\zeta^{2}\right)}{\zeta^{2} S_{k(k+1)}\left(\zeta^{2}\right)^{2}} .
$$

In a similar way, we can conjecture that the numerator in (2.4) is a polynomial in $\zeta^{2}$ of degree $n(n+1) / 2$,

$$
H_{n}^{\prime} H_{n+1}-H_{n+1}^{\prime} H_{n}=V_{n(n+1) / 2}\left(\zeta^{2}\right),
$$

so that

$$
\alpha_{2 k}=\frac{V_{k(2 k+1)}\left(\zeta^{2}\right)}{\zeta S_{k^{2}}\left(\zeta^{2}\right) S_{k(k+1)}\left(\zeta^{2}\right)^{2}}, \quad \alpha_{2 k+1}=\frac{V_{(k+1)(2 k+1)}\left(\zeta^{2}\right)}{\zeta S_{k(k+1)}\left(\zeta^{2}\right) S_{(k+1)^{2}}\left(\zeta^{2}\right)} .
$$

Increasing $n$, the complexity of expressions for $\alpha_{n}$ and $\beta_{n}$ increases quite rapidly. On the other side, using the Chebyshev algorithm, similarly as in [7], an efficient numerical construction of recursion coefficients can be done.

According to $\bar{\mu}_{k}=(-1)^{k} \mu_{k}, k \in \mathbb{N}_{0}$ (see (2.2)) we can prove: 
LEMMA 2.2. If the sequence of monic orthogonal polynomials $\left\{\pi_{n}\right\}_{n \in \mathbb{N}_{0}}$ exists, then $\pi_{n}(z)=(-1)^{n} \overline{\pi_{n}(-\bar{z})}$.

\section{Differential-difference relations}

In this section we consider some differential relations for (monic) polynomials $\left\{\pi_{n}\right\}_{n \in \mathbb{N}_{0}}$ orthogonal with respect to the linear functional (1.1), i.e., to the the (quasi) inner-product product

$$
(p, q):=\mathcal{L}[p q]=\int_{-1}^{1} p(x) q(x) w(x) d x
$$

supposing such parameters $\lambda$ and $\zeta$, which provide the existence of our orthogonal polynomials.

Our weight function $w(x)=\left(1-x^{2}\right)^{\lambda-1 / 2} e^{i \zeta x}$ satisfies the following Pearson type differential equation

$$
(\phi w)^{\prime}=\psi w, \quad \phi=1-x^{2}, \quad \psi=-(2 \lambda+1) x+i \zeta\left(1-x^{2}\right) .
$$

As before, we put $d \mu(x)=w(x) d x$.

TheOREM 3.1. For every $n \in \mathbb{N}$, we have

$$
\phi \pi_{n}^{\prime}+\psi \pi_{n}=\sum_{k=n-1}^{n+2} \theta_{n}^{k} \pi_{k}
$$

where

$$
\begin{aligned}
\theta_{n}^{n+2} & =-i \zeta, \\
\theta_{n}^{n+1} & =\zeta\left(\alpha_{n+1}+\alpha_{n}\right)-(n+2 \lambda+1), \\
2 \theta_{n}^{n} & =-(2 \lambda+1) i \alpha_{n}+i \zeta\left(1-\beta_{n+1}-\beta_{n}+\alpha_{n}^{2}\right), \\
\theta_{n}^{n-1} & =(n-1) \beta_{n},
\end{aligned}
$$

and $\alpha_{n}$ and $\beta_{n}$ are coefficients in the recurrence relation (2.3).

Proof. Since

$$
\int_{-1}^{1}\left(\phi w \pi_{n}\right)^{\prime} x^{k} d x=\left.\phi w \pi_{n} x^{k}\right|_{-1} ^{1}-k \int_{-1}^{1} \phi \pi_{n} x^{k-1} d \mu=-k \int_{-1}^{1} \phi \pi_{n} x^{k-1} d \mu=0
$$

for $k<n-1$, in that case we conclude that $\left(\phi w \pi_{n}\right)^{\prime} / w$ is orthogonal to $x^{k}$. Therefore, we have directly

$$
\frac{\left(\phi w \pi_{n}\right)^{\prime}}{w}=\phi \pi_{n}^{\prime}+\psi \pi_{n}=\sum_{k=n-1}^{n+2} \theta_{n}^{k} \pi_{k}
$$

according to the fact that $\operatorname{deg}(\psi)=2$. Since we are concerned with the monic polynomials it is readily seen that $\theta_{n}^{n+2}=-i \zeta$. 

we get

For $\theta_{n}^{n-1}$, after multiplying (3.2) with $\pi_{n-1}$ and integrating with respect to $d \mu$,

$$
\theta_{n}^{n-1}\left\|\pi_{n-1}\right\|^{2}=\int_{-1}^{1}\left(\phi w \pi_{n}\right)^{\prime} \pi_{n-1} d x=-\int_{-1}^{1} \phi w \pi_{n} \pi_{n-1}^{\prime} d x=(n-1)\left\|\pi_{n}\right\|^{2},
$$

and accordingly $\theta_{n}^{n-1}=(n-1) \beta_{n}$.

We also have

$$
\begin{aligned}
\theta_{n}^{k}\left\|\pi_{k}\right\|^{2}+\theta_{k}^{n}\left\|\pi_{n}\right\|^{2} & =\int_{-1}^{1}\left[\left(\phi w \pi_{n}\right)^{\prime} \pi_{k}+\left(\phi w \pi_{k}\right)^{\prime} \pi_{n}\right] d x \\
& =\int_{1}^{1}\left[-\phi \pi_{n} \pi_{k}^{\prime}+\phi \pi_{k}^{\prime} \pi_{n}+\psi \pi_{k} \pi_{n}\right] d \mu=\int_{1}^{1} \psi \pi_{n} \pi_{k} d \mu .
\end{aligned}
$$

The last equality for $k=n+1$ gives

$$
\begin{aligned}
\theta_{n}^{n+1} & =-\frac{\theta_{n+1}^{n}}{\beta_{n+1}}+\frac{1}{\left\|\pi_{n+1}\right\|^{2}} \int_{-1}^{1}\left[-(2 \lambda+1) x+i \zeta\left(1-x^{2}\right)\right] \pi_{n} \pi_{n+1} d \mu \\
& =-(n+2 \lambda+1)-\frac{i \zeta}{\left\|\pi_{n+1}\right\|^{2}} \int_{-1}^{1} x^{2} \pi_{n} \pi_{n+1} d \mu \\
& =-(n+2 \lambda+1)+\left(\alpha_{n+1}+\alpha_{n}\right) \zeta
\end{aligned}
$$

because of $x \pi_{n}=\pi_{n+1}+i \alpha_{n} \pi_{n}+\beta_{n} \pi_{n-1}$ and

$$
\begin{aligned}
\int_{-1}^{1} x^{2} \pi_{n} \pi_{n+1} d \mu & =\int_{-1}^{1}\left(\pi_{n+1}+i \alpha_{n} \pi_{n}+\beta_{n} \pi_{n-1}\right)\left(\pi_{n+2}+i \alpha_{n+1} \pi_{n+1}+\beta_{n+1} \pi_{n}\right) d \mu \\
& =i \alpha_{n+1}\left\|\pi_{n+1}\right\|^{2}+i \alpha_{n} \beta_{n}\left\|\pi_{n}\right\|^{2} .
\end{aligned}
$$

Similarly, for $k=n$, we get

$$
\begin{aligned}
2 \theta_{n}^{n} & =\frac{1}{\left\|\pi_{n}\right\|^{2}} \int_{-1}^{1} \psi \pi_{n}^{2} d \mu \\
& =\frac{1}{\left\|\pi_{n}\right\|^{2}}\left\{-(2 \lambda+1) \int_{-1}^{1} x \pi_{n}^{2} d \mu+i \zeta\left(\left\|\pi_{n}\right\|^{2}-\int_{-1}^{1} x^{2} \pi_{n}^{2} d \mu\right)\right\} \\
& =i\left[-(2 \lambda+1) \alpha_{n}+\zeta\left(1-\beta_{n+1}-\beta_{n}+\alpha_{n}^{2}\right)\right],
\end{aligned}
$$

because of

$$
\begin{aligned}
\int_{-1}^{1} x^{2} \pi_{n}^{2} d \mu & =\int_{-1}^{1}\left(\pi_{n+1}+i \alpha_{n} \pi_{n}+\beta_{n} \pi_{n-1}\right)^{2} d \mu \\
& =\left\|\pi_{n+1}\right\|^{2}-\alpha_{n}^{2}\left\|\pi_{n}\right\|^{2}+\beta_{n}^{2}\left\|\pi_{n-1}\right\|^{2} .
\end{aligned}
$$

Thus, the equality (3.1) is proved.

LEMma 3.1. The monic polynomials orthogonal with respect to the linear functional (1.1) satisfy the following differential-difference equation

$$
\phi \pi_{n}^{\prime}+\psi \pi_{n}=p_{2}^{n} \pi_{n}+q_{1}^{n} \pi_{n-1}, \quad n \in \mathbb{N},
$$

where $p_{2}^{n}$ and $q_{1}^{n}$ are polynomials of the second and first degree, respectively. 
Proof. Starting with equation (3.1) and using the three-term recurrence relation (2.3) we get

$$
\begin{array}{r}
\phi \pi_{n}^{\prime}+\psi \pi_{n}=\left[\left(\theta_{n}^{n+2}\left(x-i \alpha_{n+1}\right)+\theta_{n}^{n+1}\right)\left(x-i \alpha_{n}\right)-\beta_{n+1} \theta_{n}^{n+2}+\theta_{n}^{n}\right] \pi_{n} \\
+\left[-\beta_{n}\left(\theta_{n}^{n+2}\left(x-i \alpha_{n+1}\right)+\theta_{n}^{n+1}\right)+\theta_{n}^{n-1}\right] \pi_{n-1} .
\end{array}
$$

What is left to do is to read the respective polynomials.

THEOREM 3.2. The monic polynomials orthogonal with respect to the linear functional (1.1) satisfy the following equation

$$
\phi \pi_{n}^{\prime}=p_{1}^{n} \pi_{n}+q_{1}^{n} \pi_{n-1},
$$

where

$$
\begin{aligned}
& p_{1}^{n}=-n x-\frac{i \zeta}{2}\left(1-\beta_{n+1}+\beta_{n}+\alpha_{n}^{2}\right)+\frac{i \alpha_{n}}{2}(2 n+2 \lambda+1), \\
& q_{1}^{n}=\beta_{n}\left[2(n+\lambda)+i \zeta\left(x+i \alpha_{n}\right)\right] .
\end{aligned}
$$

Proof. Using expressions given in the proof of Lemma 3.1 and Theorem 3.1 we get exactly what is stated.

THEOREM 3.3. The polynomials $p_{1}^{n}$ and $q_{1}^{n}$, which appear in (3.3), satisfy the following recurrence relations

$$
\begin{aligned}
& p_{1}^{n+1}=-q_{1}^{n} \frac{x-i \alpha_{n}}{\beta_{n}}+p_{1}^{n-1}+q_{1}^{n-1} \frac{x-i \alpha_{n-1}}{\beta_{n-1}} \\
& q_{1}^{n+1}=\left(x-i \alpha_{n}\right)\left[p_{1}^{n}+q_{1}^{n} \frac{x-i \alpha_{n}}{\beta_{n}}-p_{1}^{n-1}-q_{1}^{n-1} \frac{x-i \alpha_{n-1}}{\beta_{n-1}}\right]+\phi+q_{1}^{n-1} \frac{\beta_{n}}{\beta_{n-1}},
\end{aligned}
$$

respectively.

Proof. We can prove this result using almost the same arguments from the proof in [7]. Namely, we have

$$
\begin{aligned}
\phi \pi_{n+1}^{\prime}-\phi \pi_{n}= & \left(x-i \alpha_{n}\right) \phi \pi_{n}^{\prime}-\beta_{n} \phi \pi_{n-1}^{\prime} \\
= & \left(x-i \alpha_{n}\right)\left(p_{1}^{n} \pi_{n}+q_{1}^{n} \pi_{n-1}\right)-\beta_{n}\left(p_{1}^{n-1} \pi_{n-1}+q_{1}^{n-1} \pi_{n-2}\right) \\
= & -\left(q_{1}^{n} \frac{x-i \alpha_{n}}{\beta_{n}}-p_{1}^{n-1}-q_{1}^{n-1} \frac{x-i \alpha_{n-1}}{\beta_{n-1}}\right) \pi_{n+1} \\
& +\left\{\left(x-i \alpha_{n}\right) p_{1}^{n}+q_{1}^{n-1} \frac{\beta_{n}}{\beta_{n-1}}\right. \\
& \left.+\left(x-i \alpha_{n}\right)\left(q_{1}^{n} \frac{x-i \alpha_{n}}{\beta_{n}}-p_{1}^{n-1}-q_{1}^{n-1} \frac{x-i \alpha_{n-1}}{\beta_{n-1}}\right)\right\} \pi_{n},
\end{aligned}
$$

from where we just need to read corresponding polynomials.

In the sequel we derive a system of nonlinear recurrence relations for the recursion coefficients $\alpha_{n}$ and $\beta_{n}$, which needs a few first coefficients as starting values (see Table 1). 
THEOREM 3.4. We have

$$
p_{1}^{n+1}+p_{1}^{n}+q_{1}^{n} \frac{x-i \alpha_{n}}{\beta_{n}}=(2 \lambda-1) x-i \zeta\left(1-x^{2}\right), \quad n \in \mathbb{N} .
$$

Proof. Starting with the previous theorem we can prove that quantity

$$
p_{1}^{n+1}+p_{1}^{n}+q_{1}^{n} \frac{x-i \alpha_{n}}{\beta_{n}},
$$

is independent of $n$. Hence, using Table 1, we can prove that

$$
p_{1}^{2}+p_{1}^{1}+q_{1}^{1} \frac{x-i \alpha_{1}}{\beta_{1}}=(2 \lambda-1) x-i \zeta\left(1-x^{2}\right)
$$

and hence finish the proof.

THEOREM 3.5. The three-term recurrence coefficients satisfy the following system of nonlinear recurrence equations

$$
\begin{aligned}
\beta_{n+2}= & \beta_{n}+\alpha_{n+1}^{2}-\alpha_{n}^{2}-\frac{1}{\zeta}\left[\alpha_{n+1}(2 n+2 \lambda+3)-\alpha_{n}(2 n+2 \lambda-1)\right], \\
\alpha_{n+2}= & \frac{1}{2 \beta_{n+2}}\left[2 \alpha_{n} \beta_{n+1}+\alpha_{n+1}\left(2 \beta_{n+1}-2 \beta_{n}-\beta_{n+2}+\alpha_{n+1}^{2}-\alpha_{n}^{2}\right)\right] \\
& +\frac{1}{\zeta \beta_{n+2}}\left[\alpha_{n+1}^{2}(n+\lambda+1 / 2)-\alpha_{n+1} \alpha_{n}(n+\lambda-1 / 2)\right. \\
& \left.+2 \beta_{n+2}(n+\lambda+2)-2 \beta_{n+1}(n+\lambda)-1\right]
\end{aligned}
$$

Proof. The first equation we prove quite easily. Namely, simply compare coefficients with $x^{0}$ in (3.4) and we have the stated equation.

For the proof of the second equation we have to use the second relation given in Theorem 3.3. In the same way, comparing coefficients with the term $x^{0}$ in this relation we obtain the result.

Now, we can prove a result concerning zeros of orthogonal polynomials $\pi_{n}$.

THEOREM 3.6. The polynomials $\pi_{2 n+1}, n \in \mathbb{N}_{0}$, have only simple zeros. The even polynomials $\pi_{2 n}, n \in \mathbb{N}$, have only simple zeros except possibly one zero of the multiplicity two which is purely imaginary.

Proof. It is a trivial fact that polynomials $\pi_{n}$ and $\pi_{n-1}$ cannot have zeros in common. If $x$ is multiple zero of $\pi_{n}$, using equation $(3.3)$, it must be $q_{1}^{n}(x)=0$. However, $q_{1}^{n}$ is a polynomial of first degree and, therefore, the multiplicity of the zero can be at most two. Using a symmetry property of polynomials, we see that if $x$ is a zero of the polynomial $\pi_{n}$, then also $-\bar{x}$ is the zero of this polynomial $\pi_{n}$. Hence, we conclude that $\pi_{n}$ cannot have a multiple zero outside of the imaginary axis. Finally, according to the symmetry we conclude that if polynomial of odd degree has zero on the imaginary axis it must be of odd multiplicity. Hence, all polynomials of odd degree have only simple zeros.

We can now also state a result connected with a differential equation which solutions are polynomials orthogonal with respect to the functional (1.1). 
THEOREM 3.7. The polynomials $\pi_{n}$ orthogonal with respect to the functional (1.1) satisfy the following second order differential equation

$$
\phi q_{1}^{n} \pi_{n}^{\prime \prime}+\left(\psi q_{1}^{n}-i \zeta \beta_{n} \phi\right) \pi_{n}^{\prime}-\left[-n q_{1}^{n}-i \zeta \beta_{n} p_{1}^{n}-\frac{q_{1}^{n}\left(\hat{p}_{1}^{n} q_{1}^{n}-p_{1}^{n} \hat{q}_{1}^{n}\right)}{\phi}\right] \pi_{n}=0,
$$

where we used the notation from Theorem (3.2) and

$$
\hat{p}_{1}^{n}=-q_{1}^{n-1} / \beta_{n-1} \quad \text { and } \quad \hat{q}_{1}^{n}=p_{2}^{n-1}+\left(x-i \alpha_{n-1}\right) q_{1}^{n-1} / \beta_{n-1} .
$$

The term $\hat{p}_{1}^{n} q_{1}^{n}-p_{1}^{n} \hat{q}_{1}^{n}$ must have the factor $\phi$.

PROOF. Starting with the equation (3.3) and the three-term recurrence relation, we compute easily

$$
\phi \pi_{n-1}^{\prime}=-\frac{q_{1}^{n-1}}{\beta_{n-1}} \pi_{n}+\left(p_{1}^{n-1}+q_{1}^{n-1} \frac{x-i \alpha_{n-1}}{\beta_{n-1}}\right) \pi_{n-1}=\hat{p}_{1}^{n} \pi_{n}+\hat{q}_{1}^{n} \pi_{n-1} .
$$

Combining this equation with the one for the polynomial $\pi_{n}$, i.e. with equation 3.3 , we get

$$
\phi\left[\frac{\phi \pi_{n}^{\prime}-p_{1}^{n} \pi_{n}}{q_{1}^{n}}\right]^{\prime}=\hat{p}_{1}^{n} \pi_{n}+\hat{q}_{1}^{n} \frac{\phi \pi_{n}^{\prime}-p_{1}^{n} \pi_{n}}{q_{1}^{n}} .
$$

After some calculations we obtain the following differential equation

$$
\begin{aligned}
\phi q_{1}^{n} \pi_{n}^{\prime \prime}+ & {\left[q_{1}^{n}\left(\phi^{\prime}-p_{1}^{n}-\hat{q}_{1}^{n}\right)-\phi\left(q_{1}^{n}\right)^{\prime}\right] \pi_{n}^{\prime} } \\
+ & {\left[-\left(p_{1}^{n}\right)^{\prime} q_{1}^{n}+p_{1}^{n}\left(q_{1}^{n}\right)^{\prime}-q_{1}^{n} \frac{\hat{p}_{1}^{n} q_{1}^{n}-p_{1}^{n} \hat{q}_{1}^{n}}{\phi}\right] \pi_{n}=0 . }
\end{aligned}
$$

If we use Theorems 3.4 and 3.2, for the term with $\pi_{n}^{\prime}$ we got exactly what is stated. Similarly we have the same for the term with $\pi_{n}$.

Finally, it is clear that, since all other quantities are polynomials it must be that $q_{1}^{n}\left(\hat{p}_{1}^{n} q_{1}^{n}-p_{1}^{n} \hat{q}_{1}^{n}\right) / \phi$ is polynomial, too. Obviously it cannot be that $q_{1}^{n}$ has zeros in common with $\phi$ since it can have only purely imaginary zero.

This theorem can be used to give the following result:

THEOREM 3.8. Let $n$ be an odd integer and let $x_{\nu}^{n}, \nu=1, \ldots, n$, be simple zeros of the polynomial $\pi_{n}$. Then

$$
\frac{\pi_{n}^{\prime \prime}\left(x_{\nu}^{n}\right)}{\pi_{n}^{\prime}\left(x_{\nu}^{n}\right)}+\frac{\lambda+1 / 2}{x_{\nu}^{n}-1}+\frac{\lambda+1 / 2}{x_{\nu}^{n}+1}+i \zeta-\frac{1}{x_{\nu}^{n}+i \alpha_{n}+(2 n+2 \lambda) / i \zeta}=0,
$$

for each $\nu=1, \ldots, n$.

Proof. We start with the differential equation for polynomials $\pi_{n}$, derived in the previous theorem. We divide it with $\pi_{n}^{\prime}\left(x_{\nu}^{n}\right) \neq 0, \nu=1, \ldots, n$. This equality is fulfilled due to the fact that for odd $n$, according to Theorem 3.6, the polynomial $\pi_{n}$ has only simple zeros. Thus, by division and using the fact that $\pi_{n}\left(x_{\nu}^{n}\right)=0$, $\nu=1, \ldots, n$, we have

$$
\left(\frac{\pi_{n}^{\prime \prime}}{\pi_{n}^{\prime}}+\frac{\psi}{\phi}-\frac{i \zeta \beta_{n}}{q_{1}^{n}}\right)\left(x_{\nu}^{n}\right)=0, \quad \nu=1, \ldots, n
$$

which after small calculation becomes exactly what is stated. 
This theorem enables the computation of zeros of orthogonal polynomial $\pi_{n}$ of the high degree using technique similar as given in [10].

\section{Zero distribution}

Here we want to present some consequences of Theorem 3.8 in the light of some new results of Aptekarev and Van Assche (see [1]). Namely, provided that the sequence of polynomials $\pi_{n}$ orthogonal with respect to the functional (1.1) exists, they have proved that for $\lambda=0$ the three term recurrence coefficients satisfy the following asymptotic properties $\alpha_{n} \rightarrow 0$ and $\beta_{n} \rightarrow 1 / 4$. Actually, we can use this fact to give some interesting observations connected to the zero distribution of polynomials $\pi_{n}$. A direct consequence of the mentioned theorem is the boundness of the zeros $x_{\nu}^{n}, \nu=1, \ldots, n$, of polynomials $\pi_{n}$ uniformly in $n \in \mathbb{N}$ (see [2], [3]).

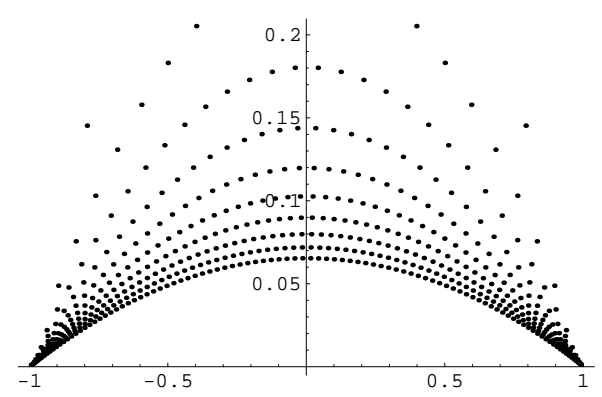

FIGURE 1. Zero distribution of $\pi_{n}, n=10(10) 100$, for $\lambda=11$ and $\zeta \approx 14.47550068655454123845163765541315197630481$ (a zero of $J_{10}$ )

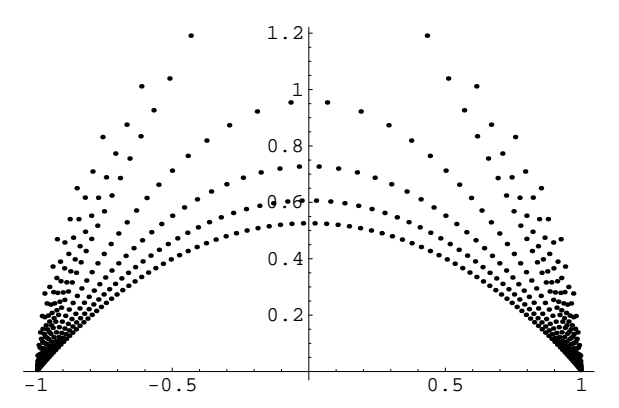

FigURE 2. Zero distribution of $\pi_{n}, n=10(10) 100$, for $\lambda=11$ and $\zeta \approx 100.71634047409274068436610723206019123535653$ (a zero of $J_{10}$ )

According to the mentioned result and for $n$ sufficiently large, our equation (3.5) becomes

$$
\frac{\pi_{n}^{\prime \prime}}{\pi_{n}^{\prime}}\left(x_{\nu}^{n}\right)+\left(\lambda+\frac{1}{2}\right)\left(\frac{1}{x_{\nu}^{n}-1}+\frac{1}{x_{\nu}^{n}+1}\right)+i \zeta+O\left(n^{-1}\right) \approx 0
$$


for each $\nu=1, \ldots, n$. If we compare it to the similar equation for the classical Gegenbauer weight $w^{0, \lambda}$ (see $[\mathbf{1 4}]$ ), we notice that there is only the difference in the term $i \zeta+O\left(n^{-1}\right)$. Taking the imaginary part we get

$$
2 \sum_{\nu \neq k} \frac{\operatorname{Im}\left(x_{k}-x_{\nu}\right)}{\left|x_{k}-x_{\nu}\right|^{2}}+\left(\lambda+\frac{1}{2}\right)\left(\frac{\operatorname{Im}\left(x_{k}\right)}{\left|x_{\nu}^{n}-1\right|^{2}}+\frac{\operatorname{Im}\left(x_{k}\right)}{\left|x_{k}^{n}+1\right|^{2}}\right)-\zeta+O\left(n^{-1}\right) \approx 0
$$

which describes a property of zeros. Namely, for a fixed $n$ and bigger $\zeta$, zeros are at the bigger distance from the $x$ axis as presented in Figures 1 and 2 .

\section{References}

[1] A. I. Aptekarev, W. V. Assche, Scalar and matrix Riemann-Hilbert approach to the strong asymptotics of Pade approximants and complex orthogonal polynomials with varying weight, J. Appox. Theory 129 (2004), 129-166.

[2] B. Beckermann, Complex Jacobi matrices, J. Comput. Appl. Math. 127 (2001), 17-65.

[3] B. Beckerman, M. Castro, On the determinacy of the comoplex Jacobi operators, Publication ANNO 446, Universite Lille, 2002.

[4] T.S. Chihara, An Introduction to Orthogonal Polynomials, Gordon and Breach, New York, 1978.

[5] A.S. Cvetković, G. V. Milovanović, The Mathematica package "OrthogonalPolynomials", Facta Univ. Ser. Math. Inform. 19 (2004), 17-36.

[6] N.I. Fel'dman, A. B. Šidlovskiŭ, The development and present state of the theory of transcendental numbers, Uspehi Mat. Nauk 22:3 (1967), (135) 3-81 (Russian) [Engl. transl. in Russian Math. Surveys 22:3 (1967), 1-79].

[7] G. V. Milovanović, A.S. Cvetković, Orthogonal polynomials and Gaussian quadrature rules related to oscillatory weight functions, J. Comput. Appl. Math. 179 (2005), 263-287.

[8] G.V. Milovanović, A.S. Cvetković, Orthogonal polynomials related to the oscillatoryChebyshev weight function, Bull. Cl. Sci. Math. Nat. Sci. Math. 30 (2005), 47-60.

[9] G. V. Milovanović, A. S. Cvetković, M. P. Stanić, Orthogonal polynomial and quadrature rules realted to Oscillatory-Gegenabuer weight function (submitted; preprint version available at: http://gauss-megatrend.edu.rs/radovi/GVM158.pdf).

[10] L. Pasquini, Accurate computation of the zeros of the generalized Bessel polynomials, Numer. Math. 86 (2000), 507-538.

[11] C. L. Siegel, Über einige Anwendungen diophantischer Approximationen, Abh. Preuss. Akad. Wiss., Phys.-Math. 1 (1929), 1-70 [Gesammelte Abhandlungen. Springer-Verlag, Berlin-New York 1966 Band I, 209-266].

[12] C. L. Siegel, Transcendental Numbers, Annals of Mathematics Studies 16, Princeton University Press, Princeton, N. J., 1949.

[13] A. B. Šidlovskiŭ, Transcendental Numbers, Nauka, Moscow, 1987 (in Russian).

[14] G. Szegő, Orthogonal Polynomials, Amer. Math. Soc. Colloq. Publ. 23, 4th ed., Amer. Math. Soc., Providence, R. I., 1975.

Faculty of Computer Sciences, Megatrend University

Beograd, Serbia

gvm@megatrend-edu.net

Faculty of Science and Mathematics, University of Niš

Niš, Serbia

aca@elfak.ni.ac.yu

Faculty of Electronic Engineering, University of Niš

Niš, Serbia

zvezdan@elfak.ni.ac.yu 